Technical Note

\title{
Damping of Sands for Varying Saturation
}

\author{
B. N. Madhusudhan ${ }^{1}$ and Jyant Kumar ${ }^{2}$
}

\begin{abstract}
A series of resonant column tests have been performed in the torsional mode of vibration to assess the effect of saturation, starting from the near dry state to the fully saturated state, on the damping ratio of sands corresponding to the threshold strain level. Tests were carried out on three different gradations of sand for various combinations of relative density and effective confining pressure. For fine sands, a certain optimum degree of saturation exists at which the damping ratio minimizes; it is known that a decrease in $S_{r}$ from a fully saturated state leads to a continuous increase in the matric suction. With an increase in the relative density, the optimum degree of saturation for fine sand increases marginally from 1.38 to $1.49 \%$, but does not show any dependency on the effective confining pressure. In contrast, the minimum values of the damping ratio for medium and coarse sands are found to always correspond to the near dry state. The damping ratio decreases continuously with increases in relative density and effective confining pressure. The threshold strain level has been found to decrease continuously with increases in relative density and effective confining pressure. DOI: 10.1061/(ASCE)GT.1943-5606.0000895. (C) 2013 American Society of Civil Engineers.
\end{abstract}

CE Database subject headings: Damping; Dynamic properties; Sand (soil type); Vibration; Saturation.

Author keywords: Damping Ratio; Dynamic Properties; Resonant column tests; Sands; Saturation.

\section{Introduction}

To determine the response of any geotechnical structure subjected to earthquake and other forms of dynamic loading, it is essential to have previous knowledge of the dynamic properties of soils and rocks. The damping ratio $(D)$ for soils reduces continuously, with decreases in strain until it reaches a threshold strain level lower than $D$, which then becomes more or less constant (Hardin and Richart 1963; Hardin and Drnevich 1972a, b; Vucetic 1994). The term damping ratio used in this paper implies a viscous damping ratio. The threshold shear strain $\left(\gamma_{t l}\right)$ is defined as the shear strain corresponding to $G / G_{o} \approx 0.99$ (Vucetic 1994), where $G$ and $G_{o}$ refer to the shear modulus corresponding to a given strain and the maximum shear modulus, respectively. The void ratio, the effective confining stress, and water content and/or degree of saturation are known to be the primary factors that significantly influence the dynamic properties of a particular soil (Hardin and Richart 1963; Hardin and Drnevich 1972a, b; Vucetic 1994; Lo Presti et al. 1997; Kumar and Madhusudhan 2010a, 2011).

The resonant column test is generally used to measure the dynamic properties for soils corresponding to a strain level in a range of $10^{-4}-10^{-1} \%$ (Chung et al. 1984; Saxena and Reddy 1989; Cascante et al. 1998; Kumar and Clayton 2007; Kumar and Madhusudhan 2010c). In the past, due to the difficulties involved in eliminating background noise and equipment-generated damping from the back electromotive force (EMF) effect, the determination of the

\footnotetext{
${ }^{1}$ Postdoctoral Research Fellow, Dept. of Civil Engineering, Univ. of Hong Kong, Hong Kong. E-mail: madhubn@ @ku.hk

${ }^{2}$ Professor, Dept. of Civil Engineering, Indian Institute of Science, Bangalore 560012, India (corresponding author). E-mail: jkumar@civil iisc.ernet.in

Note. This manuscript was submitted on June 11, 2012; approved on January 14, 2013; published online on January 16, 2013. Discussion period open until February 1, 2014; separate discussions must be submitted for individual papers. This technical note is part of the Journal of Geotechnical and Geoenvironmental Engineering, Vol. 139, No. 9, September 1, 2013. CASCE, ISSN 1090-0241/2013/9-1625-1630/\$25.00.
}

damping ratio using resonant column tests corresponding to a very small strain range $\left(10^{-4}-10^{-3} \%\right)$ had been a difficult task. At very low strain levels, the magnitude of the damping ratio of soils reaches a value that becomes comparable with the equipment-generated damping ratio. As a result, only a few damping measurements of soils could be performed successfully at very small strain levels (Cascante and Santamarina 1996; Meng and Rix 2003; Wang et al. 2003). Free vibration tests, with either no coils or with coils in an open circuit, yield almost true material damping ratio, that is, free from the counter EMF effects (Wang et al. 2003). Meng and Rix (2003) recently showed that a current mode source, with large internal resistance, rather than a commonly used voltage mode source, is capable of reducing the equipment-generated damping. Therefore, by using the current mode source, the equipment-generated damping can be minimized in modern resonant column equipment.

From the available literature, it is understood that for granular materials, especially for silts and fine sands, a certain optimum degree of saturation exists, corresponding to the maximum shear modulus (Cho and Santamarina 2001; Kumar and Madhusudhan 2011). This optimum degree of saturation has been found to increase with a decrease in the grain size of granular material (Cho and Santamarina 2001). It has been noted that the damping ratio decreases continuously with an increase in the confining pressure for sandstone rock (Winkler and Nur 1979). Winkler and Nur (1979) observed that the value of the damping ratio becomes (1) minimum for dry rock, (2) a little higher for partially saturated rock, and (3) maximum for fully saturated rock. However, very limited research seems to have been reported in the literature in which the effect of saturation has been examined on the damping ratio of sands, which provides the motive for conducting the present study (Cascante et al. 1998).

In the present paper, 198 resonant column tests have been performed in the torsion mode of vibration to assess the effect of saturation, starting from the near dry state to the fully saturated state, on the damping ratio of sands corresponding to the threshold strain level. While performing damping tests, the electric circuit through the coils is opened to ascertain a free vibration decay without back EMF generation. The effect of grain sizes, relative density, and 
effective isotropic confining pressure on the results has also been explored.

\section{Properties of Sand}

The sand was collected from the bank of the Cauvery River, located approximately $100 \mathrm{~km}$ from Bangalore, India. Depending on the range of grain sizes of the collected sand, three different sand mixtures, namely, fine, medium, and coarse, were prepared. These three different sand mixtures comprise material passing completely (1) through $425-\mu \mathrm{m}$-sieve size (fine sand mixture) but retained entirely on $75-\mu \mathrm{m}$-sieve size; (2) through $2.0 \mathrm{~mm}$ but retained on 425 - $\mu \mathrm{m}$-sieve size (medium sand mixture); and (3) 4.75-mm-sieve size but retained on 2.0-mm (coarse sand mixture), respectively (ASTM 2009). The grain size distribution curves, the specific gravity of soil solids, minimum and maximum unit weights, and the scanning electron microscope (SEM) images of the sands have already been reported in Kumar and Madhusudhan (2010a). Therefore, this information is omitted from the present paper to avoid any repetition.

\section{Sample Preparation}

To prepare a homogenous sample, the pluviation technique was adopted (Vaid and Negussey 1984; Cresswell et al. 1999); the chosen pluviation device was described in Kumar and Madhusudhan (2010a, b; 2011). The samples were prepared using a thin-wall cylindrical split mold of 50-mm internal diameter and 100-mm height. Five different relative densities $\left(D_{r}\right)$, namely, 40, 50, 60, 70, and $80 \%$ were used for performing the damping tests. The necessary calibration exercise was first performed for the dry state of the material to find the required height of fall for a given relative density (Kumar and Madhusudhan 2011). The samples associated with a given relative density were prepared in five different layers. The thickness of the each layer was kept the same. To prepare a sample with a given degree of saturation, the procedure described in Kumar and Madhusudhan (2011) was followed. For the sake of completeness, this procedure is described herein.

\section{Case 1: Partly Saturated Samples}

After correlating the height of fall with dry unit weight $\left(\gamma_{d}\right)$ of sand, the void ratio $(e)$ is determined from the expression, $e=$ $\left(G_{s} \gamma_{w} / \gamma_{d}-1\right)$, where $G_{s}$ is the specific gravity of soils solids and $\gamma_{w}$ is the unit weight of water. For a chosen relative density, to prepare a sample with a given degree of saturation $\left(S_{r}\right)$, the required water content $(w)$ is determined by using the expression $w=e S_{r} / G_{s}$. On this basis, from the known dry weight of the sample (50-mm diameter and 100-mm height), the volume of deaired water to achieve the given values of $S_{r}$ and $D_{r}$ is determined. This volume of water is then divided into five equal portions, and each part is sprinkled uniformly over each of the five layers used for preparing the sample. After completion of each resonant column test, the dimensions of the sample and the weights of the moist and oven-dried sample are measured. These data are used to find the exact values of dry unit weight $\left(\gamma_{d}\right)$, bulk unit weight $(\gamma)$, and water content $(w)$ of the sample. From known values of $\gamma_{d}, \gamma$, and $w$, the values of $D_{r}$ and $S_{r}$ are determined. For each selected value of $\sigma_{3}^{\prime}$ and $S_{r}$, a new sample is prepared by using exactly the same procedure as described earlier, in which $\sigma_{3}^{\prime}$ is the effective isotropic pressure.

\section{Case 2: Fully Saturated Samples}

After preparing a dry soil sample with a given $D_{r}$ and after attaining the magnitude of $\sigma_{3}^{\prime}$, the value of Skempton's $B$ parameter is determined. From a known value of $B$, the value of $S_{r}$ is then indirectly determined from the basic definition of the parameter $B$ (Kumar and Madhusudhan 2011). The magnitude of $B$ was kept greater than 0.99 .

\section{Testing Procedure}

The resonant column apparatus (RCA), supplied by GDS Instruments (Hampshire, United Kingdom), was used. The test sample is fixed at its base, and the drive mechanism is attached to the top of the sample. After attaining the resonant frequency in the torsion mode of vibration, the input current supply to the coils is then switched off to perform a free vibration test, and the response of the accelerometer with time is then recorded. The amplitude decay curve with time is recorded, from which the magnitude of the damping ratio is computed. The peak amplitude of each cycle is determined. The value of the logarithmic decrement, $\delta$, is then determined by the expression

$$
\delta=\frac{1}{n} \log _{e} \frac{z_{1}}{z_{1+n}}
$$

where

$$
\delta=\log _{e} \frac{z_{1}}{z_{2}}=\frac{2 \pi D}{\sqrt{1-D^{2}}}
$$

where $D=$ viscous damping ratio; $z_{1}=$ peak amplitude recorded for the first cycle; $z_{2}=$ peak amplitude recorded for the second cycle; $z_{n+1}=$ peak amplitude recorded for the $(n+1)$ th cycle; and $n=$ number of cycles.

After calculating the value of $\delta$, the value of the damping ratio can be determined by using Eq. (2). In the present study, the free vibration decay curve for the first three cycles was used to determine the value of the damping ratio. The free vibration decay test for different values of the input current to the coils was performed to determine the variation of the damping ratio with the strain level. The shear strain $(\gamma)$ is calculated from the expression given by Kumar and Clayton (2007).

To provide some assessment about the equipment-generated damping, tests were performed on aluminum calibration bars of 10-, 12.35-, and 15-mm diameter at different shear-strain levels. The measured values of the viscous damping ratios were found to vary between 0.02 and $0.05 \%$, which confirms that the equipment damping remains negligible.

\section{Test Results}

\section{Threshold Strain Level and Damping of Near Dry Sands}

The threshold shear strain refers to the value of strain below which the magnitude of the shear modulus $(G)$ becomes greater than 0.99 times the maximum shear modulus $\left(G_{o}\right)$. The values of $G_{o}$ from the resonant column tests generally compare well with the values that are measured separately using the bender element tests (Kumar and Madhusudhan 2010b; Madhusudhan 2011). The thesis of Madhusudhan (2011) can be referred to for the variation of the shear modulus with shear strain. The values of the threshold shear strains, along with corresponding values of $D_{o}$ have been provided 
exclusively in Table 1; the subscript $o$ has been used to indicate the value of $D$ corresponding to the threshold strain level. The magnitude of $D_{o}$ decreases continuously with increases in the values of effective confining pressure and relative density. The threshold shear strain remains generally greater than $1 \times 10^{-3} \%$. It can be seen that the threshold shear strain decreases continuously with increases in relative density and effective confining pressure. However, no clear trend has been observed for the variation of the threshold shear strain with respect to changes in grain sizes of sand. For the sands used in this study, the range of the threshold shear strain has been found to be (1) $3.22 \times 10^{-3}$ to $4.16 \times 10^{-3} \%$ for fine sand, (2) $3.27 \times 10^{-3}$ to $5.12 \times 10^{-3} \%$ for medium sand, and (3) $3.10 \times 10^{-3}$ to $4.31 \times 10^{-3} \%$ for coarse sand, For all three sands, the value of the threshold strain remains greater than $1 \times 10^{-3} \%$. The value of $D_{o}$ in the near dry state for different chosen sands was found to lie within a range of $0.49-2.17 \%$ for selected values of $D_{r}$ and $\sigma_{3}^{\prime}$.

Table 1. Values of Threshold Shear Strain and Corresponding Values of Damping Ratio $\left(D_{o}\right)$ in the Dry State for the Sands Used in the Study

\begin{tabular}{|c|c|c|c|c|c|c|c|}
\hline \multirow[b]{2}{*}{$\sigma_{3}^{\prime}(\mathrm{kPa})$} & \multirow[b]{2}{*}{$\begin{array}{c}\text { Relative density, } \\
D_{r}(\%)\end{array}$} & \multicolumn{2}{|c|}{ Fine sand } & \multicolumn{2}{|c|}{ Medium sand } & \multicolumn{2}{|c|}{ Coarse sand } \\
\hline & & $\begin{array}{l}\text { Threshold shear } \\
\text { strain } 10^{-3}(\%)\end{array}$ & $D_{o}(\%)$ & $\begin{array}{l}\text { Threshold shear } \\
\text { strain } 10^{-3}(\%)\end{array}$ & $D_{o}(\%)$ & $\begin{array}{l}\text { Threshold shear } \\
\text { strain } 10^{-3}(\%)\end{array}$ & $D_{o}(\%)$ \\
\hline \multirow[t]{5}{*}{100} & 40 & 4.16 & 2.17 & 5.12 & 1.73 & 4.31 & 1.70 \\
\hline & 50 & 3.90 & 2.00 & 4.70 & 1.65 & 4.09 & 1.57 \\
\hline & 60 & 3.63 & 1.80 & 3.93 & 1.57 & 3.84 & 1.43 \\
\hline & 70 & 3.44 & 1.71 & 3.63 & 1.47 & 3.65 & 1.35 \\
\hline & 80 & 3.34 & 1.64 & 2.90 & 1.32 & 3.35 & 1.26 \\
\hline \multirow[t]{5}{*}{300} & 40 & 4.07 & 1.56 & 4.29 & 1.39 & 3.88 & 1.37 \\
\hline & 50 & 3.95 & 1.41 & 3.98 & 1.25 & 3.70 & 1.26 \\
\hline & 60 & 3.66 & 1.13 & 3.71 & 1.03 & 3.62 & 1.13 \\
\hline & 70 & 3.62 & 1.07 & 3.64 & 0.94 & 3.42 & 0.96 \\
\hline & 80 & 3.50 & 0.77 & 3.58 & 0.86 & 3.28 & 0.76 \\
\hline \multirow[t]{5}{*}{500} & 40 & 3.66 & 1.08 & 3.86 & 1.12 & 3.83 & 1.15 \\
\hline & 50 & 3.59 & 0.92 & 3.72 & 1.09 & 3.66 & 0.99 \\
\hline & 60 & 3.49 & 0.68 & 3.53 & 0.90 & 3.34 & 0.83 \\
\hline & 70 & 3.31 & 0.62 & 3.35 & 0.76 & 3.20 & 0.73 \\
\hline & 80 & 3.22 & 0.49 & 3.27 & 0.52 & 3.10 & 0.50 \\
\hline
\end{tabular}
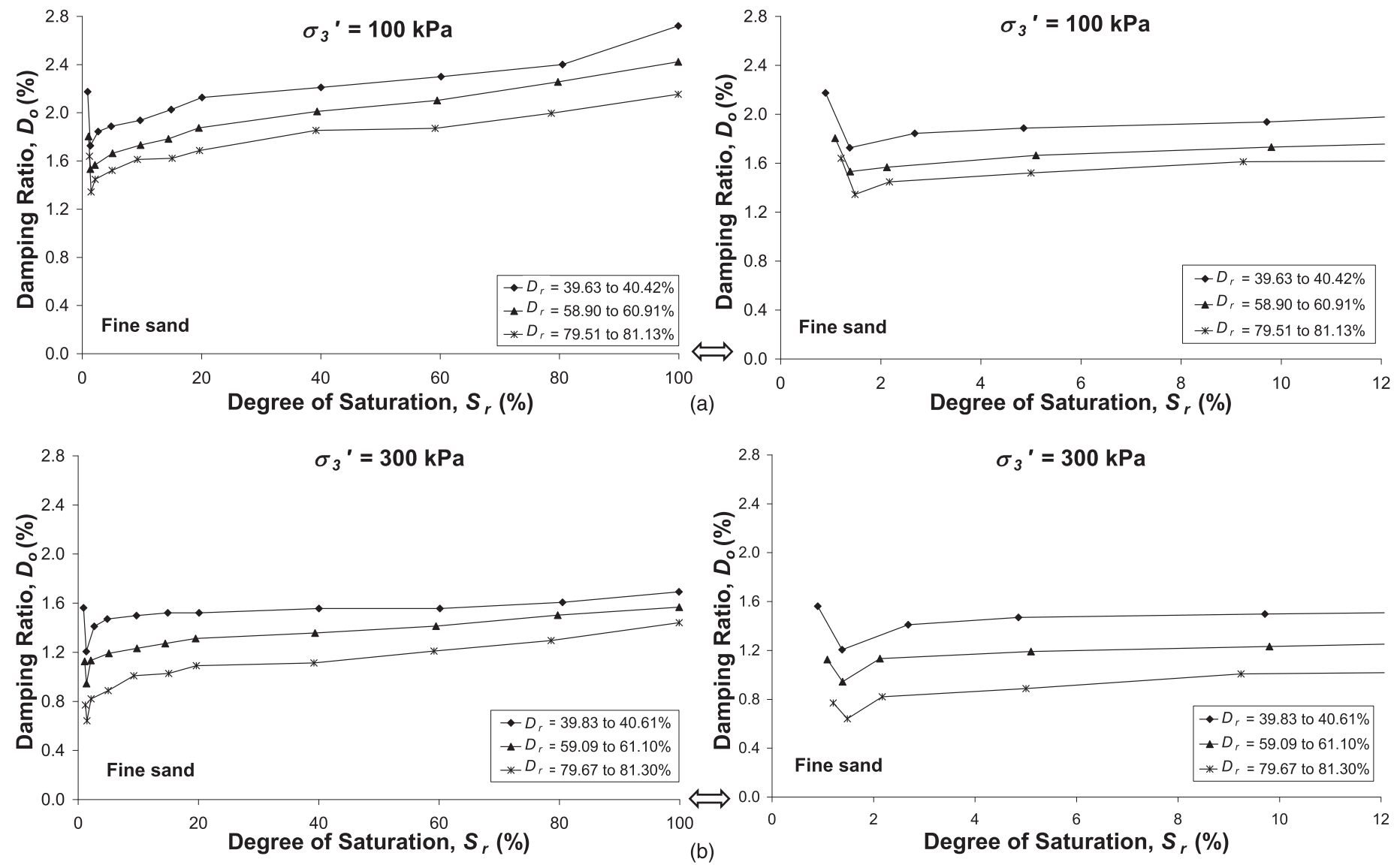

$\sigma_{3}^{\prime}=300 \mathrm{kPa}$

Fig. 1. Variation of the damping ratio with $S_{r}$ for fine sand at different relative densities for (a) $\sigma_{3}^{\prime}=100 \mathrm{kPa}$; (b) $\sigma_{3}^{\prime}=300 \mathrm{kPa}$ 

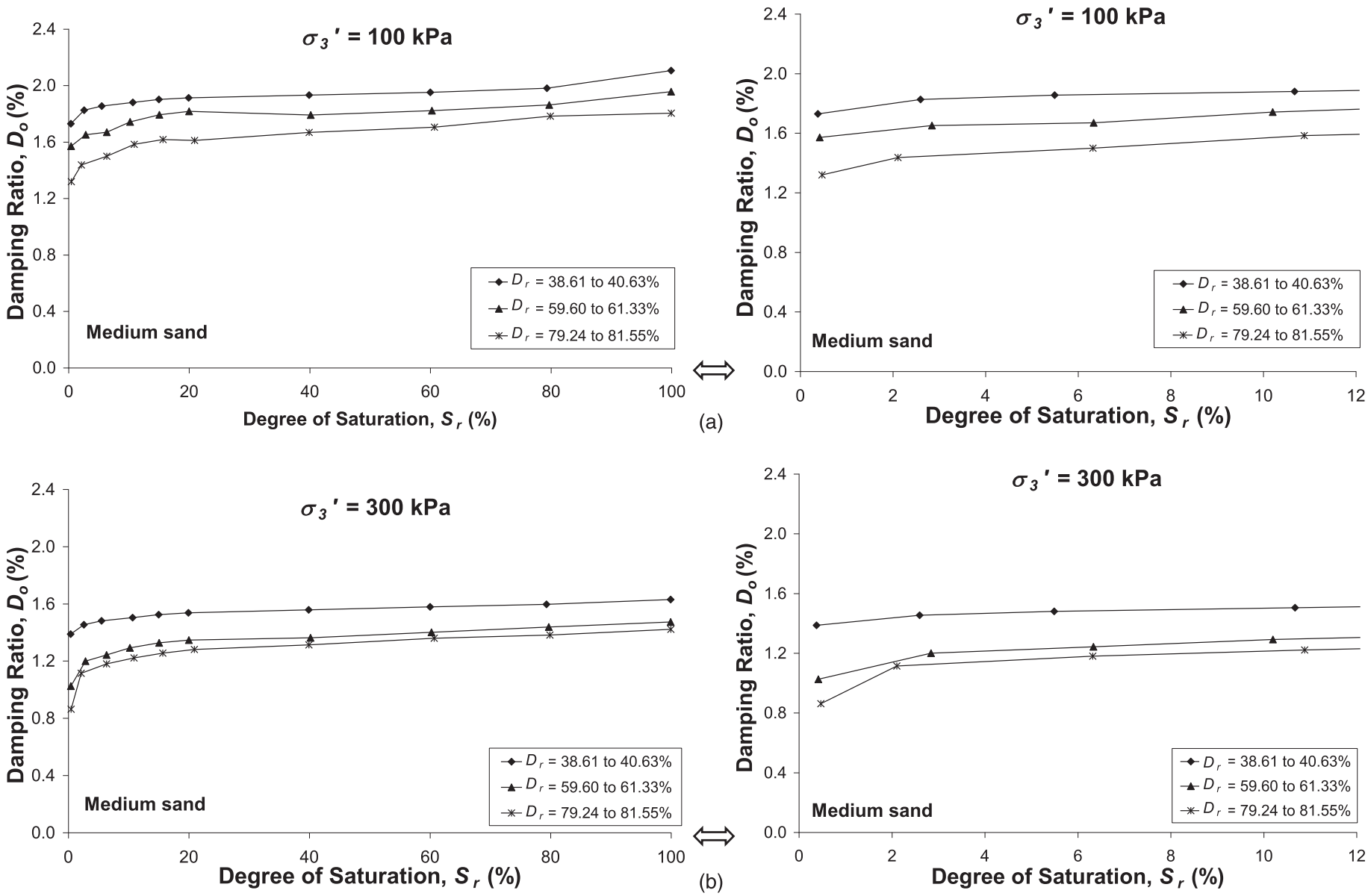

Fig. 2. Variation of the damping ratio with $S_{r}$ for medium sand at different relative densities for (a) $\sigma_{3}^{\prime}=100 \mathrm{kPa}$; (b) $\sigma_{3}^{\prime}=300 \mathrm{kPa}$

\section{Effect of Saturation on Damping Ratio of Sands}

The damping ratios studied in this paper, for different degrees of saturations, have been evaluated corresponding to the threshold shear strain. The variation of the damping ratio $\left(D_{o}\right)$, with changes in degree of saturation for different values of relative density and confining pressure for all three sands, are presented in Figs. 1-3. For fine sand, the damping ratio becomes minimum, corresponding an optimum value of $S_{r}$; the minimum value of $D_{o}$ is referred to as $D_{o \text {-opt }}$. For $S_{r}$ greater than the optimum value, the magnitude of $D_{o}$ increases continuously, with an increase in $S_{r}$ until the soil becomes fully saturated, in which case the damping ratio attains the maximum value. The minimum values of $D_{o}$ and the associated degree of saturation for all three sands, for different combinations of $D_{r}$ and $\sigma_{3}^{\prime}$ have been provided in Table 2. For the fine sand, the optimum degree of saturation varies in a narrow range of $1.38-1.49 \%$. The value of the optimum degree of saturation increases marginally with an increase in the relative density of the sample, whereas a variation in $\sigma_{3}^{\prime}$ does not bring any change in the optimum degree of saturation. The minimum degree of saturation is between 0.38 and $0.47 \%$ for the medium sand and between 0.10 and $0.13 \%$ for the coarse sand. For all three sands, the minimum value of $D_{o}$ decreases with increases in $D_{r}$ and $\sigma_{3}^{\prime}$. For values of $S_{r}$ smaller than the optimum value, a sudden rise occurs in the value of $D_{o}$ with a decrease in $S_{r}$ for fine sand. For the medium and coarse sands, the minimum value of $D_{o}$ has been found to be associated with the smallest $S_{r}$ value (the near dry state) that can be achieved experimentally. It should be noted that the present observations related to the effect of saturation on the damping ratio are similar to those reported by Cho and Santamarina (2001) and Kumar and Madhusudhan (2011) with respect to the effect of the variation of $S_{r}$ on the shear modulus of sands. At the optimum degree of saturation, the magnitude of the shear modulus becomes the maximum (Madhusudhan 2011). The values of the optimum degree of saturation reported in Madhusudhan (2011), with respect to the variation of $G_{o}$ with $S_{r}$ for these sands, are exactly the same as given in the present study. The results associated with the variation of the shear modulus with degree of saturation have been exclusively provided in Madhusudhan (2011). These results are, therefore, not reported herein.

Hypothesis for the Observed Effect of $S_{\boldsymbol{r}}$ on the Damping Ratio A decrease in $S_{r}$ from a fully saturated state leads to an increase in the matric suction and the effective stress on the sample. Consequently, starting from $S_{r}=100 \%$, with a decrease in $S_{r}$, a continuous increase in the shear modulus and a reduction in the damping ratio are observed. The increasing matric suction with a further decrease in $S_{r}$ causes the air to enter into the pore water medium. The corresponding state of the water phase has been referred to as the funicular regime by Cho and Santamarina (2001). Matric suction and the effective confining pressure in the funicular regime increases with a reduction in $S_{r}$. With a further reduction in $S_{r}$, a pendular regime finally occurs, and the water phase becomes disconnected (Cho and Santamarina 2001). Matric suction at this stage, however, acts only at the contact between particles (wherever water droplets exist), and it exhibits a significant increase because of the small radii of the water 

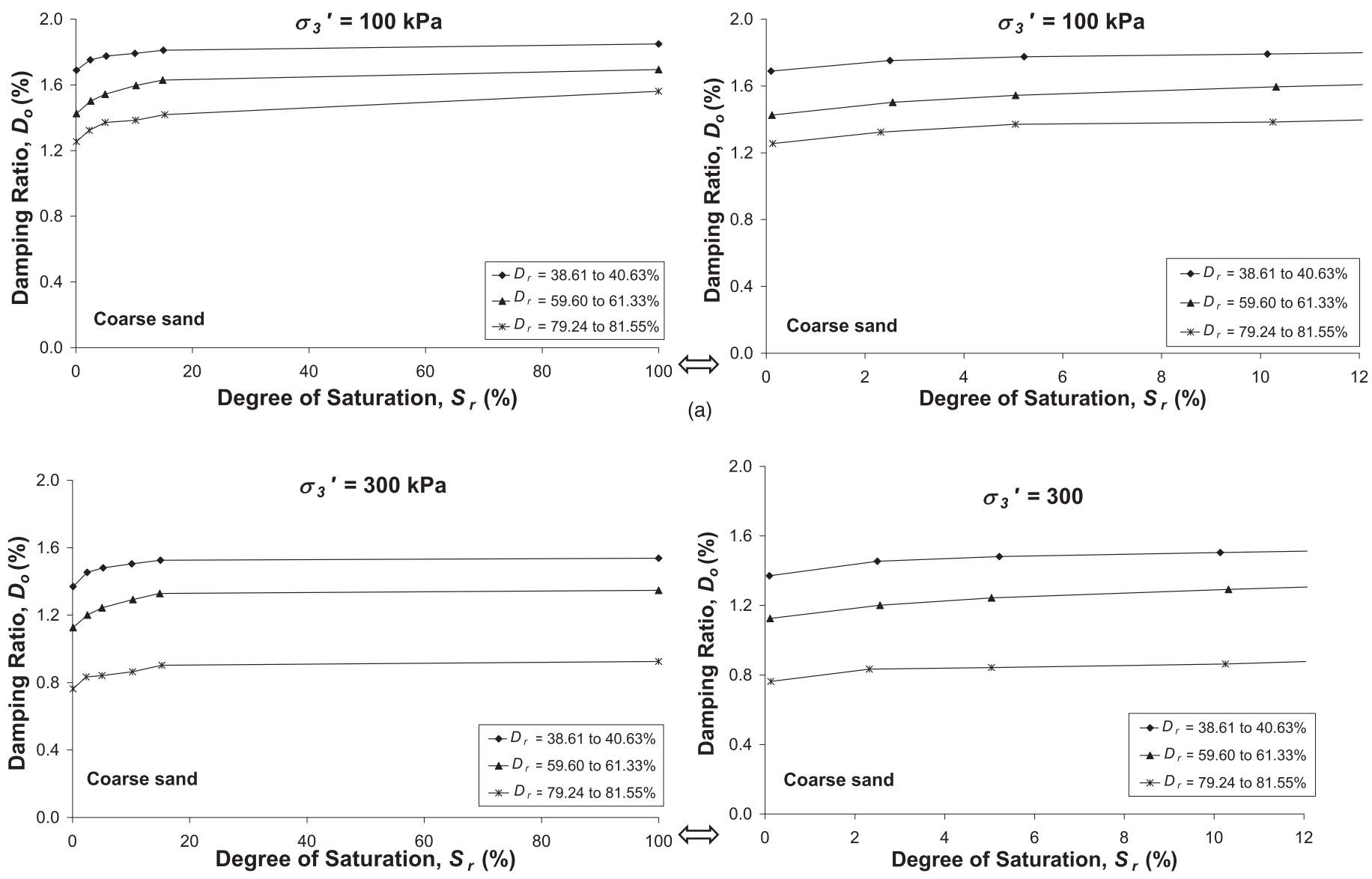

(b)

Fig. 3. Variation of the damping ratio with $S_{r}$ for coarse sand at different relative densities for (a) $\sigma_{3}^{\prime}=100 \mathrm{kPa}$; (b) $\sigma_{3}^{\prime}=300 \mathrm{kPa}$

Table 2. Minimum Values of $D_{o}$ and the Associated Degree of Saturation for Different Values of Relative Density $\left(D_{r}\right)$ and Effective Confining Pressure $\left(\sigma_{3}^{\prime}\right)$

\begin{tabular}{|c|c|c|c|c|c|c|c|}
\hline \multirow[b]{2}{*}{$\sigma_{3}^{\prime}(\mathrm{kPa})$} & \multirow[b]{2}{*}{$\begin{array}{c}\text { Relative } \\
\text { density, } D_{r}(\%)\end{array}$} & \multicolumn{2}{|c|}{ Fine sand } & \multicolumn{2}{|c|}{ Medium sand } & \multicolumn{2}{|c|}{ Coarse sand } \\
\hline & & $\begin{array}{c}\text { Optimum degree of } \\
\text { saturation }(\%)\end{array}$ & $D_{o \text {-opt }}(\%)$ & $\begin{array}{c}\text { Optimum degree of } \\
\text { saturation }(\%)\end{array}$ & $D_{o \text {-opt }}(\%)$ & $\begin{array}{c}\text { Optimum degree of } \\
\text { saturation }(\%)\end{array}$ & $D_{o-\text { opt }}(\%)$ \\
\hline \multirow[t]{5}{*}{100} & 40 & 1.38 & 1.72 & 0.38 & 1.73 & 0.10 & 1.70 \\
\hline & 50 & 1.38 & 1.64 & 0.39 & 1.65 & 0.11 & 1.57 \\
\hline & 60 & 1.40 & 1.53 & 0.42 & 1.57 & 0.12 & 1.43 \\
\hline & 70 & 1.43 & 1.48 & 0.44 & 1.47 & 0.12 & 1.35 \\
\hline & 80 & 1.49 & 1.34 & 0.47 & 1.32 & 0.13 & 1.26 \\
\hline \multirow[t]{5}{*}{300} & 40 & 1.38 & 1.20 & 0.38 & 1.39 & 0.10 & 1.37 \\
\hline & 50 & 1.38 & 1.06 & 0.39 & 1.25 & 0.11 & 1.26 \\
\hline & 60 & 1.40 & 0.94 & 0.42 & 1.03 & 0.12 & 1.13 \\
\hline & 70 & 1.43 & 0.77 & 0.44 & 0.94 & 0.12 & 0.96 \\
\hline & 80 & 1.49 & 0.64 & 0.47 & 0.86 & 0.13 & 0.76 \\
\hline \multirow[t]{5}{*}{500} & 40 & 1.38 & 0.88 & 0.38 & 1.12 & 0.10 & 1.15 \\
\hline & 50 & 1.38 & 0.78 & 0.39 & 1.09 & 0.11 & 0.99 \\
\hline & 60 & 1.40 & 0.57 & 0.42 & 0.90 & 0.12 & 0.83 \\
\hline & 70 & 1.43 & 0.53 & 0.44 & 0.76 & 0.12 & 0.73 \\
\hline & 80 & 1.49 & 0.43 & 0.47 & 0.52 & 0.13 & 0.50 \\
\hline
\end{tabular}

menisci. However, the overall effective area covered by the water droplets over a certain cross-sectional area will decrease with a reduction in $S_{r}$. As a result, the net effective stress on the sample may decrease after a certain value of $S_{r}$ (termed the optimum degree of saturation), which will result in an increase in the damping ratio and a reduction of the shear modulus of the sample. With a decrease of
$S_{r}$, as observed from the present experimental data, $S_{r}$ is less than the optimum value for fine sand. Nonexistence of the optimum degree of saturation for medium and coarse sands can be the result of experimental difficulties in attaining very low values of $S_{r}$ while performing the resonant column tests. However, more research is needed in the future to examine whether any optimum degree of saturation exists in 
the case of medium and coarse sands, and to provide clarification(s) for the observed minimum damping ratio and/or maximum shear modulus at the optimum degree of saturation.

\section{Conclusions}

By carrying out a series of resonant column tests in the torsion mode of vibration, the effect of saturation has been examined on the damping ratio of sands associated with the threshold strain level for different combinations of grain size range, relative density, and effective confining pressure. The damping ratio decreases invariably with increases in the values of $D_{r}$ and $\sigma_{3}^{\prime}$. The value of the threshold strain, associated with $G / G_{o}=0.99$, has been found to reduce marginally with increases in the values of $D_{r}$ and $\sigma_{3}^{\prime}$. For fine sands, a certain optimum degree of saturation always exists at which the damping ratio minimizes, and simultaneously, at which the shear modulus always maximizes. The optimum degree of saturation does not change with $\sigma_{3}^{\prime}$ but increases marginally with an increase in $D_{r}$. For medium and coarse sands, the minimum damping ratio is always associated with the near dry state. The damping ratio reaches the maximum value for fully saturated sands, regardless of the grain size. With an increase in $S_{r}$, from the near dry to the fully saturated state, the maximum change in the damping ratio is generally between 0.5 and $1 \%$. This indirectly implies that the damping variations with saturation may not be really necessary to be considered if the dynamic behavior of sand has to be modeled.

\section{Acknowledgments}

The financial support provided by the Department of Science and Technology, India, under the project "Dynamic properties of cohesionless soils using resonant column and bender element tests and their effects on ground response amplification," is gratefully acknowledged.

\section{References}

ASTM. (2009). "Standard practice for description and identification of soils (visual-manual procedure)." D 2488-09, West Conshohocken, PA.

Cascante, G., and Santamarina, J. C. (1996). "Interparticle contact behavior and wave propagation." J. Geotech. Eng., 122(10), 831-839.

Cascante, G., Santamarina, C., and Yassir, N. (1998). "Flexural excitation in a standard torsional-resonant column." Can. Geotech. J., 35(3), 478-490.
Cho, G. C., and Santamarina, J. C. (2001). "Unsaturated particulate materials-particle level studies.” J. Geotech. Geoenviron. Eng., 127(1), 84-96.

Chung R. M., Yokel F. Y., and Drnevich V. P. (1984). "Evaluation of dynamic properties of sands by resonant column testing." Geotech. Testing J., $7(2), 60-69$.

Cresswell, A., Barton, M. E., and Brown, R. (1999). "Determining the maximum density of sands by pluviation." Geotech. Testing J., 22(4), 324-328.

Hardin, B. O., and Drnevich, V. P. (1972a). "Shear modulus and damping in soils measurement and parameter effects." J. Soil Mech. Found. Div., 98(6), 603-624.

Hardin, B. O., and Drnevich, V. P. (1972b). "Shear modulus and damping in soils: Design equation and curves." J. Soil Mech. Found. Div., 98(7), 667-691.

Hardin, B. O., and Richart, F. E., Jr. (1963). "Dissipation of elastic wave energy in granular soils." J. Soil Mech. Found. Div., 89(6), 27-56.

Kumar, J., and Clayton, C. R. (2007). "Effect of sample torsional stiffness on resonant column test results." Can. Geotech. J., 44(2), 221-230.

Kumar, J., and Madhusudhan, B. N. (2010a). "Effect of relative density and confining pressure on Poisson ratio from bender and extender elements tests." Geotechnique, 60(7), 561-567.

Kumar, J., and Madhusudhan, B. N. (2010b). "A note on the measurement of travel times using bender and extender elements." Soil. Dyn. Earthquake Eng., 30(7), 630-634.

Kumar, J., and Madhusudhan, B. N. (2010c). "On determining elastic modulus of a cylindrical sample subjected to flexural excitation in resonant column apparatus." Can. Geotech. J., 47(11), 1288-1298.

Kumar, J., and Madhusudhan, B. N. (2011). "Dynamic properties of sand from dry to fully saturated states." Geotechnique, 62(1), 45-54.

Lo Presti, D. C. F., Jamiolkowski, M., Pallara, O., Cavallaro, A., and Pedroni, S. (1997). "Shear modulus and damping of soils." Geotechnique, 47(3), 603-617.

Madhusudhan, B. N. (2011). "Dynamic properties of dry to fully saturated states using resonant column and bender element tests." Ph.D. thesis, Indian Institute of Science, Bangalore, India.

Meng, J., and Rix, G. J. (2003). "Reduction of equipment generated damping in resonant column measurements." Geotechnique, 53(5), 503-512.

Saxena, K. S., and Reddy, K. R. (1989). "Dynamic moduli and damping ratios for Monterey No. 0 sand by resonant column tests." Soils Found., 29(2), 37-51.

Vaid, Y. P., and Negussey, D. (1984). "Relative density of pluviated sand samples." Soils Found., 24(2), 101-105.

Vucetic, M. (1994). "Cyclic threshold shear strains in soils." J. Geotech. Eng., 120(12), 2208-2228.

Wang, Y. H., Cascante, G., and Santamarina, J. C., (2003). "Resonant column testing: the inherent counter EMF effect." Geotech. Testing J., 26(3), 342-352.

Winkler, K., and Nur, A. (1979). "Pore fluids and seismic attenuation in rocks." Geophys. Res. Lett., 6(1), 1-4. 